\title{
Stimulus saliency and anchoring: Temporal and end stimulus effects'
}

\author{
JAMES BIERI AND KAY KUJALA, UNIVERSITY OF TEXAS \\ ALVIN L, ATKINS, YESHIVA UNIVERSITY
}

Two characteristics considered to influence the saliency of an anchor stimulus are its natural end stimulus quality and its temporal relation to the target stimulus. Using physical and social continua which differed in these respects, greater anchoring effects were observed when more salient anchors were used.

Because the magnitude of anchoring effects should be directly related to the saliency of anchor stimuli, factors influencing such stimulus saliency warrant greater empirical study. Extreme stimuli of dimensions do differ in terms of their natural end-anchor qualities (Bieri et al, 1966). Johnson \& King (1964) demonstrated the effect of more salient extreme stimuli along a physical continuum and Miller \& Bieri (1965) found that less salient end stimuli affected relative discriminability within both physical and social continua. A second factor that should affect saliency of an anchor stimulus is the temporal relation between it and the other stimuli being judged. It would be expected that greater contrast effects in judgment would be observed when the time interval between anchor stimulus and target stimulus is minimal, as when simultaneous presentation is used. Conversely, reduced contrast effects would be expected when an interval of time elapses between presentation of the anchor and target stimulus. In this latter instance opportunity for direct comparison is eliminated and memory decay would add to the less potent effect of the prior anchor stimulus.

By varying both the relative strength of an extreme (anchor) stimulus and the temporal relation between anchor and target stimulus, we would expect greater contrast effects when a more natural end stimulus was used in simultaneous presentation and less pronounced anchoring effects when a weak natural end stimulus was used in sequential presentation. The present study analyzes the influence of inese stimulus saliency factors in an anchoring task in which alternate end stimuli of a dimension are used as anchor stimuli. Such an alternation of anchor method has been found to induce initial contrast effects followed by subsequent assimilation effects (Bieri, Orcutt, \& Leaman, 1963; Bieri et al, 1966; Atkins, in press).

\section{Method}

Variation in saliency of end-stimulus anchors was achieved by using two stimulus dimensions, one social (aggression behavior) and one physical (size of squa res). If, as expected (Miller \& Bieri, 1965), end stimuli of a behavioral dimension such as aggression were more natural end stimuli, then judgments of the extreme stimuli on such a continuum should be less variable than the judgments of the extreme stimuli on a continuum of size. To verify these expectations, a group of 52 Ss judged six times a series of 15 behavioral items ranging from low aggression to high aggression. Another group of $87 \mathrm{Ss}$ made the same number of judgments of 15 sizes of squares, ranging from 2 in. to $5-1 / 2$ in. Variability of judgment of the two extreme stimuli and the middle stimulus, assessed by the range of judgments, is presented in Fig. 1.

Judgments of aggression stimuli differed significantly from judgments of squares $(F=36.18, p<.005)$. Differences between stimuli were also significant $(F=59.37$, $\mathrm{p}<.005)$, as was the interaction between dimensions and stimuli $(F=20.37, p<.005)$. That the interaction effect derived primarily from differences in judgment of extreme stimuli is apparent in Fig. 1. There is, then, striking evidence for the greater saliency of end stimuli for the aggression dimension than for the size dimension.

The anchoring task was given to $250 \mathrm{Ss}$ in eight conditions described below. For Ss judging squares, end anchor stimuli were two 2-in. squares or two 5-1/2-in. squares presented in context with the middle-size square (3-3/4 in.). For Ss judging aggressive stimuli, anchor stimuli were two extreme high or two extreme low aggression items. For the alternation of anchor design, Ss first received two extreme anchor stimuli in context with a middle stimulus (trial 1), and then two anchor stimuli from the opposite end of the continuum with a middle stimulus (trial 2). This

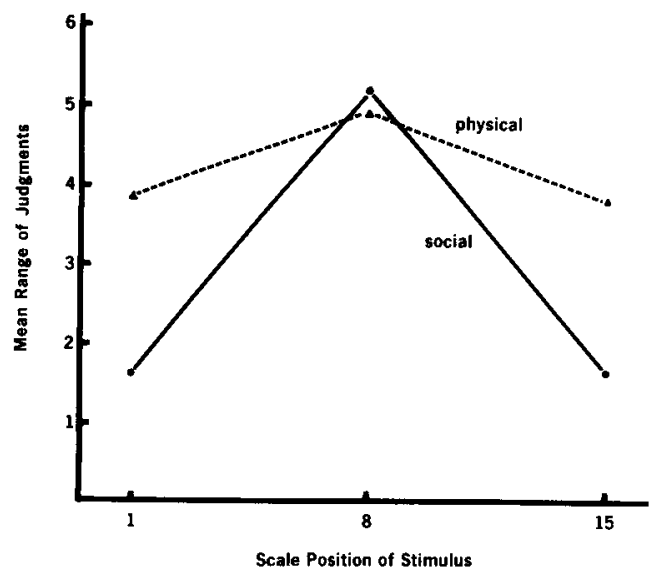

Fig. 1. Mean range of judgments of extreme and middle stimuli. 
alternation continued for five trials. For those Ss judging social stimuli, different middle and extreme stimuli with comparable pre-scaled aggression values were used for each trial. Within each stimulus domain, approximately one half of the Ss had initial high anchors and one half initial low anchors. Judgments were made on a 15-interval scale such that 15 represented high aggression or large size.

To vary saliency temporally, approximately one half of the Ss were presented the two anchor stimuli and the middle stimulus simultaneously while judgments of all three were made. The other Ss were presented single stimuli in sequence, such that the first anchor stimulus was presented, removed, and judged, followed by the second anchor stimulus and the target (middle) stimulus. In this manner, all Ss made a total of 15 judgments.

\section{Resulis and Discussion}

Figure 2 presents the anchoring curves of middle stimulus judgments for each of the four conditions within the social and physical stimulus realms. Analyzing trial 1 judgments first, for which contrast effects were expected, it can be observed that for both social and physical stimuli, simultaneous stimulus presentation produced greater contrast effects than did single

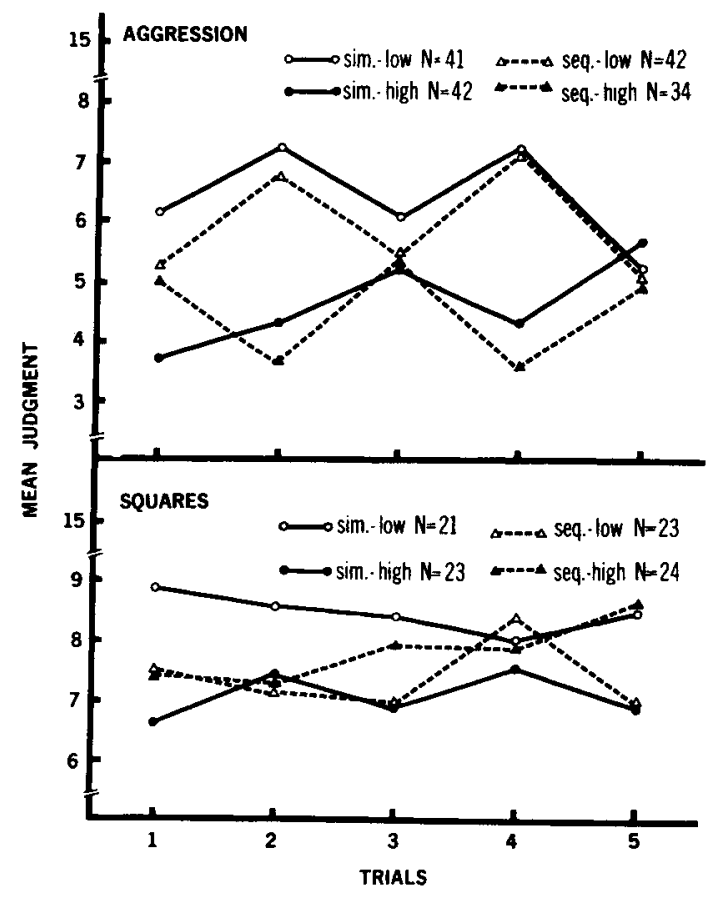

Fig. 2. Mean judgment of middle social and physical stimuli with simultaneous (sim.) and sequential (seq.) presentation for initial high and low anchor groups. (sequential) stimulus presentation. For social stimuli, the significance between the two temporal conditions in amount of contrast effect is significant $(t=1.99$, $p<.05)$ while for physical stimuli the difference does not reach significance $(t=1.67, p<.10)$. In terms of initial contrast effects, the expected greater contrast with simultaneous compared to sequential presentation did occur. Further, this difference between temporal conditions was significant for the social stimuli, which had the most salient end stimuli as anchors.

Turning to the effects of stimulus saliency upon trials 2 to 5 , the most apparent difference between the curves in Fig. 2 is the substantially greater assimilation effects with social stimuli than with physical stimuli. Using a trial to trial criterion of assimilation (Bieri et al, 1963) such that a shift in judgment toward the anchor (e.g., from trial 1 to trial 2) reflects assimilation, then for social stimuli 15 of the 16 shifts are in the assimilation direction. Chi-square $=4.17$ for these distribution differences $(p<.05)$. Further, it is apparent from Fig. 2 that when assimilation occurred for physical stimuli, the shifts toward the anchor stimulus were of lower magnitude than for social stimuli.

The results help clarify the unsettled nature of assimilation effects noted in the literature (Bieri et al, 1966). Only one of the nine assimilation shifts on subsequent trials for physical stimuli, and the sole contrast shift for social stimuli, occurred with simultaneous presentation. Apparently, assimilation effects are least likely when direct comparison of the target stimulus with the anchor stimulus is possible. Further, the greater assimilation trends for social stimuli also may be a function of the fact that while the same middle physical stimulus was present on all trials, each middle social stimulus was different. Assimilation could be facilitated when anchor stimuli are strong natural end stimuli and when moderate stimuli are most ambiguous.

\section{References}

Atkins, A. L. Own attitude and discriminability in relation to anchoring effects in judgment. J. Pers. soc. Psychol., in press.

Bieri, J., Atkins, A. L., Briar, S., Leaman, R. L., Miller, H., \& Tripodi, T. Clinical and social judgment. New York: Wiley, 1966.

Bieri, J., Orcutt, B. A., \& Leaman, R. Anchoring effects in sequential clinical judgments. J. abnorm. soc. Psychol., 1963, 67, 616-623.

Johnson, D. M., \& King, C. R. Systematic study of end anchoring and central tendency of judgment. J. exp. Psychol., 1964, 67, 501-506.

Miller, H., \& Bieri, J. End anchor effects in the discriminability of physical and social stimuli. Psychon. Sci., 1965, 3, 339-340.

\section{Note}

1. This research was supported by grant GS-842 from the National Science Foundation and P. H. S. fellowship MH-30, 398-01 from the National Institute of Health. 